Thorax, 1977, 32, 1-4

\title{
Mycobacterium tuberculosis resistance to rifampicin and ethambutol: a clinical survey
}

\author{
J. B. MACDONALD ${ }^{1}$
}

From Sully Hospital, Sully, South Glamorgan

\begin{abstract}
Macdonald, J. B. (1977). Thorax, 32, 1-4. Mycobacterium tuberculosis resistance to rifampicin and ethambutol: a clinical survey. Ninety-seven cultures of Myco. tuberculosis referred to the Tuberculosis Reference Laboratory, Cardiff during 1972-73 and found to be resistant to rifampicin or ethambutol were studied. Resistance to each drug appeared equally common. Few lower grades of rifampicin resistance occurred, cultures tending to be either fully sensitive or highly resistant. Intermediate grades of ethambutol resistance, however, were common, and a majority of highly resistant cultures were preceded by cultures showing lower grades of resistance. Case-notes were obtained on 84 patients $(87 \%)$ and revealed no cases of primary resistance. In the three years since first developing resistance $36 \%$ of patients died: in the majority tuberculosis was a major cause of death. Half the patients had been prescribed unsatisfactory drug regimens containing rifampicin or ethambutol by their physicians, and only a small minority were considered to have co-operated well. One-third had discharged themselves from hospital against medical advice and only $21 \%$ attended clinics regularly. These two factors probably accounted for the development of resistance in most cases.
\end{abstract}

The two antituberculosis drugs, rifampicin and ethambutol, are now widely used in first-line chemotherapy (British Medical Journal, 1973). The present study reviews 84 patients resistant to one or both of these drugs.

\section{Method}

Cultures of Mycobacterium tuberculosis may be referred to the Tuberculosis Reference Laboratory, Cardiff, from any laboratory in England and Wales for sensitivity testing. Sensitivities are assessed by the method of Leat and Marks (1970). All cases of resistance to rifampicin and ethambutol in cultures referred during 1972-73 were noted, excluding cases showing borderline resistance on one occasion only. The mode of evolution of resistance was studied. Requests were then sent for the hospital and chest clinic case-notes of these patients. The case-notes were analysed to discover any possible cases of primary resistance.

When resistance was felt to be of the acquired type the patients' tuberculosis history and social circumstances were noted. To study the quality

'Present address: City Hospital, Hucknall Road, Nottingham of treatment, drug doses and the use of sensitivity testing were analysed. The minimum chemotherapy regarded as sufficient was 15 months of treatment with adequate doses of two antituberculosis drugs to which the patient was known not to be resistant. Minimum acceptable dosages for streptomycin were $0.75 \mathrm{~g}$ daily or twice weekly, for PAS $10 \mathrm{~g}$ daily, for isoniazid $200 \mathrm{mg}$ daily or $12 \mathrm{mg} / \mathrm{kg}$ twice weekly, for ethambutol $15 \mathrm{mg} /$ $\mathrm{kg}$ daily, and for rifampicin $450 \mathrm{mg}$ daily. In regimens inadequate in several respects, only the most severe failing was noted.

Three criteria were used for minimum use of drug sensitivity testing. First, testing should be requested after six months' persistently positive sputum smears during treatment or on recurrence of positive smears after two or more negative smears on treatment. Sensitivities should be requested during the next three months or after two outpatient visits (which ever is the longer period). Secondly, testing should be performed before changes of regimen are made or within three months afterwards. Thirdly, when testing shows a new resistance the drug should be removed from the regimen within three months or after two visits if the organism is resistant or highly resist- 
ant, or the test should be repeated if a lower grade of resistance is found.

Patient cooperation was assessed subjectively from case-notes and objectively by noting selfdischarge from either hospital or clinic and by failure to attend at least one-quarter of outpatient clinic appointments. Medical insight into patient cooperation was also assessed.

\section{Results}

\section{CULTURES}

A total of 97 cases of Myco. tuberculosis resistance to rifampicin or ethambutol was found in 1972-73. The Table shows that rifampicin resistance remained static in $1972-73$ but that ethambutol resistance rose from 31 cases in 1972 to 44 cases in 1973. Forty-four per cent of the 97 cases

Table Cultures referred in 1972 and 1973: degrees of resistance shown to rifampicin and enthambutol (97 cases)

\begin{tabular}{|c|c|c|c|c|c|c|c|}
\hline \multirow{2}{*}{ Drug } & \multirow{2}{*}{ Year } & \multicolumn{5}{|c|}{ Resistance (no. of cases) } & \multirow{2}{*}{ Total } \\
\hline & & $\boldsymbol{H R}$ & $\boldsymbol{R}$ & $R R 4$ & $R R 3$ & $\boldsymbol{B}$ & \\
\hline Rifampicin & $\begin{array}{l}1972 \\
1973 \\
\text { Total }\end{array}$ & $\begin{array}{l}25 \\
31 \\
56\end{array}$ & $\begin{array}{l}4 \\
1 \\
5\end{array}$ & $\begin{array}{l}1 \\
0 \\
1\end{array}$ & $\begin{array}{l}2 \\
2 \\
4\end{array}$ & $\begin{array}{l}0 \\
0 \\
0\end{array}$ & $\begin{array}{l}32 \\
34 \\
66\end{array}$ \\
\hline Ethambutol & $\begin{array}{l}1972 \\
1973 \\
\text { Total }\end{array}$ & $\begin{array}{l}14 \\
23 \\
37\end{array}$ & $\begin{array}{l}2 \\
0 \\
2\end{array}$ & $\begin{array}{l}10 \\
15 \\
25\end{array}$ & $\begin{array}{l}1 \\
1 \\
2\end{array}$ & $\begin{array}{l}4 \\
5 \\
9\end{array}$ & $\begin{array}{l}31 \\
44 \\
75\end{array}$ \\
\hline
\end{tabular}

HR = highly resistant; $R=$ resistant; RR4 = resistant to ratio 4; $R R 3=$ resistant to ratio 3; $B=$ borderline. For details of the procedure see Leat and Marks (1970).

showed resistance to both drugs. The pattern of resistance differed between ethambutol and rifampicin. Lower resistance grades (resistant $-\mathbf{R} 4$, resistant $-\mathbf{R} 3$, and borderline) were common for ethambutol ( $48 \%$ ) but rare for rifampicin $(8 \%)$. Cultures tended to be either highly resistant or fully sensitive to rifampicin. Of the 37 cases highly resistant to ethambutol, $22(59 \%)$ developed resistance gradually, previous cultures showing lower grades of resistance. No case of this evolution of resistance occurred with rifampicin. One culture highly resistant to ethambutol returned to full sensitivity gradually after the drug was stopped.

\section{PATIENTS}

Adequate case-notes were obtained in 84 of the 97 cases $(87 \%)$. All cases had pulmonary tuberculosis and no case of primary resistance was found. Sixty-four were male. At the time of the first resistant culture patients had had tuberculosis for a mean of 10.3 years and the mean age was 55 years. Nearly all patients had been born $\frac{\bar{c}}{\bar{N}}$ in the British Isles with only four from the Indian $\frac{\vec{\sigma}}{\overrightarrow{0}}$ subcontinent. Most men were heavy smokers but 을 a majority of women did not smoke. Two-thirds $ळ$ of the men drank at least three pints of beer per $\overrightarrow{0}$ day, one third were either heavy drinkers or overt $\vec{\longrightarrow}$ alcoholics. Only two women were alcoholics and $\vec{\omega}$ three were moderate drinkers. Fifty-six per cent of subjects came from social classes 4 and 5 . $\times$ Only $23 \%$ of men and $30 \%$ of women had jobs, $\stackrel{\omega}{\sim}$ homes, and caring relatives. Over half the patients had severe social problems. Nine men and one woman were vagrant.

Thirty-eight patients had other relevant diseases such as peptic ulcer, pneumoconiosis or diabetes. Twelve patients were taking corticosteroids. In three cases they were medically necessary ( $2 \stackrel{2}{2}$ Addison's disease and one severe asthma) but in $\overrightarrow{0}$ the remaining nine steroids were prescribed as general tonics. In the mean period of three years between the first resistant culture and the study 30 of 84 patients died (36\%). All had active tuberculosis at death and in half this was the sole cause of death.

\section{QUALITY OF MEDICAL CARE}

Quality of medical care involves three aspects. First, was the prescribed regimen adequate to cure a cooperative patient? Secondly, was drug sensitivity testing used adequately? Thirdly, did the physician perceive and cope adequately with any lack of patient cooperation?

\section{REGIMEN ADEQUACY}

Rifampicin or ethambutol was used as initial therapy in $14 \%$ of cases. In the other $86 \%$ an initial regimen was changed to include rifampicin or ethambutol as 'reserve' drugs. The indication for change was proven resistance to one or more initial drugs $(50 \%)$, assumed resistance $(30 \%)$, drug reaction $(2 \%)$, or unknown $(4 \%)$. The initial regimen had been inadequate in 45 of 73 cases $(62 \%)$, because of inadequate dosage $(23 \%)$, too short a course $(26 \%)$, or the use of only one drug $(12 \%)$. The regimens containing rifampicin or ethambutol were inadequate in 44 of 84 cases $(52 \%)$, because of inadequate dosage $(20 \%)$, too short a course $(8 \%)$, or inadequate cover with effective drugs $(24 \%)$.

\section{SENSITIVITY TESTING}

Sensitivity testing was not performed promptly with persistent or recurrent sputum positivity 
during treatment in 54 patients (64\%). Sputum sensitivity was not tested on at least one change of regimen in 55 patients $(65 \%)$. Relevant sensitivity testing results were ignored in 44 patients $(52 \%)$. The regimens of 22 patients $(26 \%)$ were changed to include a drug to which a previous culture had been highly resistant when other effective drugs were still available. General practitioners interfered with regimens in 11 cases, of which five were the direct result of misleading letters from physicians. Dispensing chemists altered regimens to give inadequate doses in two cases.

\section{PATIENT COOPERATION}

Regularity of drug taking is difficult to assess. From case-note comments it appears that $20 \%$ of patients were very uncooperative throughout, $30 \%$ moderately uncooperative, $26 \%$ reasonably cooperative, and $15 \%$ fully cooperative. Seven mildly demented patients could not cooperate. The more objective method of documenting cooperation failure showed that $33 \%$ of patients had discharged themselves from hospital against medical advice. Nineteen per cent of subjects had discharged themselves from outpatient care and $60 \%$ of patients failed to attend at least one in every four outpatient appointments. Only 18 patients $(21 \%)$ attended outpatient follow-up regularly.

\section{MEDICAL INSIGHT INTO COOPERATION}

The behaviour of 71 patients showed that they were very unlikely to be cooperating fully. In 25 uncooperative cases $(35 \%)$ physicians seemed unaware of any cooperation failure. In 23 cases $(32 \%)$ they appeared partially aware and in 23 cases $(32 \%)$ the physicians seemed totally aware of their patients' failings. Cooperation could be improved by persuasion, by attempts to solve social problems, and by the use of supervised chemotherapy. In 30 cases $(42 \%)$ no effort was made to improve cooperation. In 20 cases $(28 \%)$ limited attempts were made and in 21 cases $(30 \%)$ the physician tried hard to improve cooperation.

\section{URINE TESTING}

Outpatient urine testing is an important practical method for monitoring patient drug taking objectively. Urine testing is available for rifampicin (Eidus and Harnanansingh, 1969) and ethambutol (Eidus et al., 1971). Only three of 84 patients had urine testing recorded at every visit (all patients at the same clinic), 13 patients $(15 \%)$ had occasional urine testing, and the other $68(81 \%)$ never had any urine tested for drugs.

\section{SUPERVISED CHEMOTHERAPY}

Twenty-four of 71 uncooperative patients had been given supervised chemotherapy, paramedical staff giving drugs under direct supervision either twiceor thrice-weekly. This method had succeeded in 13 cases $(54 \%)$. The case-notes of the 47 uncooperative patients not given supervised chemotherapy were assessed. They suggested that a supervised regimen would have been successful with normal supervision in 12 cases $(26 \%)$ and with unusually painstaking supervision in 21 patients $(45 \%)$ and that it would have failed in 14 patients.

\section{Discussion}

The wider use of rifampicin and ethambutol will produce more resistant cases. In our reference laboratory cultures, resistance appears equally frequent to either drug. The abrupt change found in rifampicin resistance from fully sensitive to highly resistant confirms the genetic studies of Tsukamura (1972) showing a single phenotype of rifampicin resistance. This feature makes rifampicin resistance easier to measure reliably.

In contrast, ethambutol resistance evolved gradually. Thus a report of lower grade ethambutol resistance should be taken seriously, enabling an earlier remedy of treatment failures. The development of resistance during chemotherapy could be due to inadequate medical care or to poor patient cooperation. Medical care is based on using acceptable regimens. Well over half our patients had inadequate regimens of both initial and reserve drugs. Drug sensitivity testing was used inadequately and the results were ignored in a majority of cases. These disquieting figures suggest a substantial role for inadequate management in producing drug-resistant tuberculosis. They accord with the finding of the British Thoracic and Tuberculosis Association (1971) that 87 of 140 patients who died from tuberculosis after at least three months' treatment had been prescribed inadequate chemotherapy. Both general practitioners and chemists interfered with therapy. Better communication should reduce this problem and that of the use of corticosteroids as general tonics. Treatment continuity will suffer if drug-resistant patients are seen in clinics by a rapid succession of registrars. This happened in one-quarter of the cases. Complex drug-resistant cases should be referred automatically to senior staff. Poor patient cooperation is difficult to assess fully. It appeared to be a dominant factor in a majority of patients who acquired drug resistance. 
Physicians often appeared unaware of this problem, even after repeated failure to attend for follow-up. Cooperation failure can be detected more easily if clinic urines are routinely tested for drugs. This was not done in $81 \%$ of cases. Supervised chemotherapy was successful in the majority of cases in which it was tried and would have been successful in two-thirds of the remainder. It deserves a larger role in the management of drugresistant cases.

The present study suggests that on discovering drug resistance of the acquired type the physician should first scrutinise his therapy. If an excellent regimen has been prescribed and the patient is obtaining his tablets correctly the patient must be failing to take his therapy regularly. Despite the plausibility of the protests this is the only tenable conclusion. Then supervised chemotherapy should be the rule rather than the exception.

The largest single element in producing acquired resistance is lack of patient cooperation. However, medical mismanagement plays an uncomfortably large role. If physicians abided by the well-tried rules of antituberculosis chemotherapy a sizeable portion of acquired drug resistance would not occur.

I should like to thank Dr. J. Marks, Dr. A.
Seaton, and Dr. E. Macdonald for encouragement and helpful criticism and Miss P. Edwards for secretarial assistance.

\section{References}

British Medical Journal (1973). Rifampicin or $\vec{\circ}$ ethambutol in the routine treatment of tuberculosis (Editorial). British Medical Journal, 4, 568.

British Thoracic and Tuberculosis Association (1971). A survey of tuberculosis mortality in England and Wales in 1968. Tubercle, 52, 1-17.

Eidus, L. and Harnanansingh, A. M. T. (1969). Simple procedures for checking rifampicin in urine. American Review for Respiratory Disease, 100, 음 738-739.

Eidus, L., Harnanansingh, A. M. T., and Jessamine, A. G. (1971). Test for detection of ethambutol in urine. Canadian Journal of Public Health, 62, 251252.

Leat, J. L. and Marks, J. (1970). Improvement of drug-sensitivity tests on tubercle bacilli. Tubercle, 51, 68-75.

Tsukamura, M. (1972). The pattern of resistance development to rifampicin in Mycobacterium tuberculosis. Tubercle, 53, 111-117.

Requests for reprints to: Dr. J. B. Macdonald, City Hospital, Hucknall Road, Nottingham. 